\title{
Prostatic leiomyoma in a young man
}

\author{
A M Abeygunasekera ${ }^{1}$, M S G R Kumara ${ }^{1}$, A M L C Ambegoda ${ }^{1}$, D D Ranasingha ${ }^{2}$, C P Sosai ${ }^{3}$
}

Ceylon Medical Journal 2019; 64: 76-77

DOI: http://doi.org//10.4038/cmj.v64i2.8893

Isolated solid lesions in the prostate are rare in young men and pose a diagnostic and therapeutic dilemma. Management should be radical enough to prevent recurrences if it is to be a neoplasm, while it should not entail over-treatment causing functional derangements like urinary incontinence, retrograde ejaculation and erectile dysfunction.

A previously healthy 35-year old man with slow urinary stream was found to have an asymmetrically enlarged left prostatic lobe which was firm and having a smooth surface. His serum PSA was $1.1 \mathrm{ng} / \mathrm{ml}$. Transrectal ultrasound scan (TRUS) revealed a mass in the left prostatic lobe containing solid and cystic areas. MRI scan showed a well-defined solid lesion with multiple cystic areas in the left lobe of prostate (Figure 1). It was low in signal intensity in T1W images and showed heterogenous signal intensity in T2W images. Lesion was surrounded by a hypointense halo with no evidence of infiltration. TRUS guided prostate biopsy revealed a benign spindle cell tumour. Since the lesion showed benign features both in MRI and biopsy and considering his age, transurethral resection of the mass was done rather than a more radical excision.
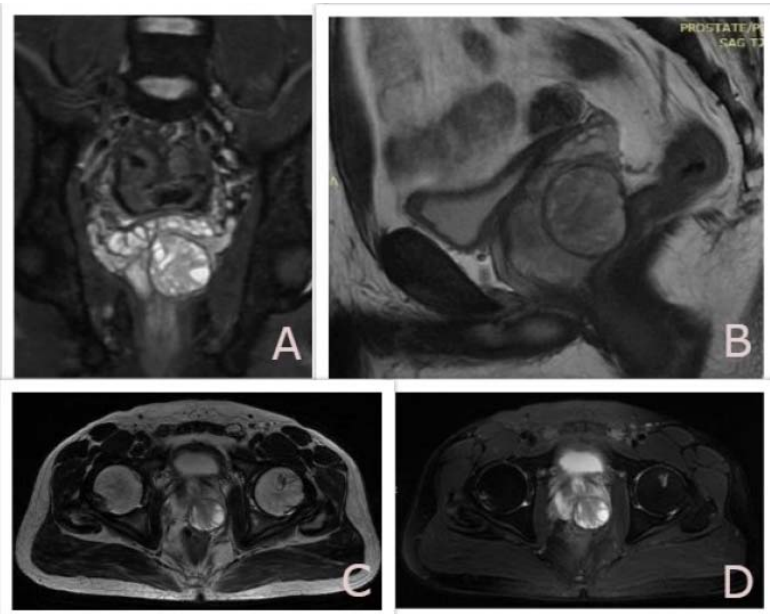

Figure 1. MRI images showing the lesion in the left lobe of the prostate (A) coronal T2 (B) sagittal T2 (C) axial T2, and (D) axial T2 FS views.
The histology of the resected tissue showed cytologically bland spindle cells arranged in interlacing fascicles in a myxoid stroma infiltrated by lymphocytes, plasma cells, neutrophils and mast cells. There were no glandular tissues seen. Immunohistochemical staining showed desmin and SMA positivity and CD34 negativity confirming a diagnosis of leiomyoma of prostate (Figure 2).
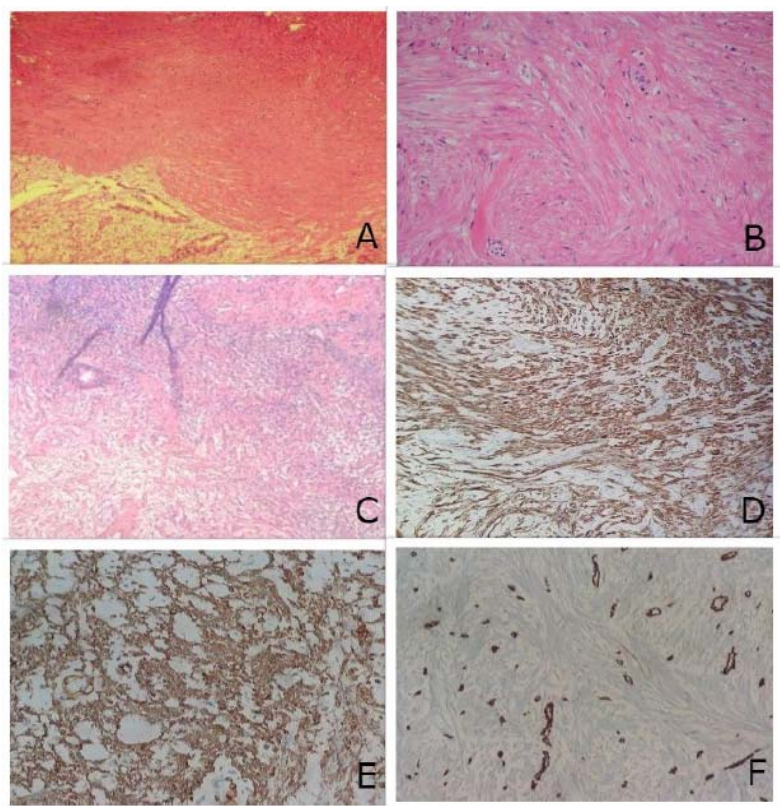

Figure 2. Microscopic images (A) H\&E stain ( $\times$ 100), (B) H\&E stain ( $\times 400$ ), (C) myxoid stroma H\&E ( $\times 100)(D)$ Desmin positivity ( $\times 400)$, (E) SMA positivity ( $\times 400)$, and (F) CD34 negativity $(\times 400)$.

Prostatic leiomyoma is a rare benign smooth muscle tumour known to occur in older patients [1]. The reported cases so far have an average age around 60 years and this patient is the youngest to have a leiomyoma indicating its possibility even in thirties [1-4]. It should be differentiated from nodular leiomyomatous stromal hyperplasia and

${ }^{1}$ Department of Urology, Colombo South Teaching Hospital, Kalubowila, ${ }^{2}$ Department of Radiology, Colombo South Teaching Hospital, Kalubowila, ${ }^{3}$ Department of Pathology, Colombo South Teaching Hospital, Kalubowila, Sri Lanka.

Correspondence: AMA, e-mail: <amabey@sltnet.lk>. Received 19 March 2019, revised version received 9 June 2019 and accepted 16 June 2019. 
leiomyosarcoma [2]. Leiomyoma is devoid of glandular tissue and has a capsule when compared to nodular leiomyomatous stromal hyperplasia which occurs in advanced benign prostatic hyperplasia. Leiomyoma can be differentiated from leiomyosarcoma, by its low cellularity, circumscription and lack of cytological atypia and mitosis. Serum PSA has been normal in all patients with leiomyoma reported so far similar to this patient.

Leiomyoma shows a homogenous sign after gadolinium administration in MRI [3]. Images can be diverse related to the amount of degeneration within the lesion. Immunohistocemistry confirms the diagnosis as leiomyoma is positive for desmin and actin while negative for CD 34, CD 117, S 100 and cytokeratin [3,4]. Presence of multinucleate giant cells with vacuoles constitute atypical cells [2]. The treatment offered in previous cases include transurethral resection, open retropubic prostatectomy, radical prostatectomy and even cystoprostatectomy with ileal conduit formation [1-4]. Although most authors worry about sarcomatous transformation such an eventuality has not been reported even with cases of leiomyomata with atypia [1].

\section{Conflict of interests}

Authors declare that there are no conflict of interests.

\section{References}

1. Hossain D, Meiers I, Qian J, Machennan GT, Bostwick DG. Prostatic leiomyoma with atypia: follow-up study of 10 cases. Ann Dign Pathol 2008; 12: 328-32.

2. Barba J, Tolosa E, Panizo AF, Beriam JM. Prostatic leiomyoma, case report. Arch Esp Urol 2011; 64: 631-5.

3. Ringoir A, Rappe B, Dhaene K, Schallier D. Prostatic leiomyoma: A case report. Urol Case Rep 2016; 9: 457.

4. Kapp B, Abarzua-Cabezas F, Cusano A, Meraney A. An unusually large leiomyoma of the prostate. Can J Urol 2014; 21: 7157-9. 